\title{
Estudo retrospectivo de cães portadores de ruptura do ligamento cruzado cranial: 32 casos (2006 a 2012)
}

\section{A retrospective study of dogs with cranial cruciate ligament rupture: 32 cases (2006-2012)}

\author{
Madalena Bach ${ }^{1}$; José Ademar Villanova Junior ${ }^{2 *}$; Ubirajara Iobe Tasqueti ${ }^{3}$ Cláudia \\ Turra Pimpão ${ }^{4}$; Antonia Maria Binder do Prado ${ }^{5}$; Pedro Vicente Michellotto Junior ${ }^{6}$
}

\begin{abstract}
Resumo
A ruptura do ligamento cruzado cranial (RLCCr) é uma das lesões mais comuns no cão e a maior causa de doença articular degenerativa (DAD) do joelho. Os animais não tratados exibem DAD dentro de poucas semanas e alterações graves dentro de poucos meses. A gravidade da degeneração parece ser diretamente proporcional ao porte e idade. Doenças articulares inflamatórias sistêmicas são associadas com a ruptura deste ligamento. $\mathrm{O}$ objetivo deste trabalho foi caracterizar a população de cães acometidos de RLCCr segundo os seguintes fatores: tipo de claudicação, ruptura ligamentar uni ou bilateral, raça, idade, sexo, estado reprodutivo, dieta, peso corporal, presença de DAD e luxação patelar anteriores à RLCCr. A base dos dados foi a análise de prontuários de 32 cães com RLCCr no período de janeiro de 2006 a dezembro de 2012. Os resultados mostram que a maioria $(56,25 \%)$ apresentava claudicação de apoio no momento da consulta. A RLCCr unilateral foi a mais frequente $(90,62 \%), 13,8 \%$ dos casos apresentaram RLCCr no membro contralateral, após um período médio de 2 anos e 4 meses. A raça de maior ocorrência foi o Poodle toy $(21,9 \%)$ e Pitbull $(18,75 \%)$; cães sem raça definida foram responsáveis por $12,5 \%$ dos casos. As idades com maior frequência foram 2, 3, $4 \mathrm{e}$ 7 anos (média $=5,7$ anos). Com relação ao sexo e estado reprodutivo, as fêmeas $(68,75 \%)$ e animais não castrados $(69 \%)$ foram mais acometidos. Quanto à dieta, a maioria $(59,38 \%)$ ingeria dieta comercial. Em relação ao peso dos pacientes, a maioria (25\%) pesava entre 26 e $35 \mathrm{~kg}$. DAD e luxação patelar anteriores à RLCCr foram vistos em 53,12\% e 21,88\% respectivamente. Pode-se observar que os dados em nosso meio tendem a se assemelhar com a literatura consultada: ocorrência de RLCCr em animais adultos de grande porte, pesando entre 26 e $35 \mathrm{~kg}$ com ruptura unilateral e claudicação de apoio, sendo a fêmea mais representativa. Animais que recebem dieta comercial e não são castrados foram mais acometidos. Luxação patelar anterior à RLCCr foi percebida na minoria dos animais, diferentemente da DAD anterior à RLCCr, que foi vista na maioria dos casos.

Palavras-chave: Claudicação, doença articular degenerativa, osteoartrose, joelho
\end{abstract}

${ }_{1}$ Médica Veterinária, Pesquisadora, Pontifícia Universidade Católica do Paraná, PUCPR, São José dos Pinhais, PR. E-mail: madalenabach@hotmail.com

2 Prof. Dr. Adjunto I, Doutorado em Ciências Veterinárias, PUCPR, São José dos Pinhais, PR. E-mail: jose.villanova@pucpr.br

3 Prof. Assistente II, Mestrado em Ciência Animal, PUCPR, São José dos Pinhais, PR. E-mail: ubirajara.tasqueti@pucpr.br

${ }^{4}$ Prof ${ }^{a}$ Titular, Doutorado em Processos Biotecnológicos, PUCPR, São José dos Pinhais, PR. E-mail: claudia.pimpao@pucpr.br

${ }_{5}$ Prof ${ }^{a}$ Titular, Doutorado em Patologia e Cirurgia de Pequenos Animais, PUCPR, São José dos Pinhais, PR. E-mail: antonia. prado@pucpr.br

${ }_{6}^{6}$ Prof. Titular, Doutorado em Biologia Celular e Molecular, PUCPR, São José dos Pinhais, PR. E-mail: michelottojunior@gmail. com

* Autor para correspondência 


\begin{abstract}
Rupture of the cranial cruciate ligament (RLCCr) is one of the most common injuries in the dog and the leading cause of degenerative joint disease (DAD) of the knee. Untreated animals exhibit degenerative joint changes in a few weeks and severe changes within a few months. The severity of degeneration appears to be directly proportional to body size and age. Systemic inflammatory joint diseases are associated with the rupture of this ligament. The aim of this study was to characterize the population of dogs suffering from RLCCr according to the following factors: type of lameness, unilateral or bilateral ligament rupture, breed, age, sex, reproductive status, diet, body weight, presence of DAD and patellar luxation prior to RLCCr. The data base was created from information from medical records of 32 dogs diagnosed with RLCCr in Companion Animals Hospital Uninity, Catholic University of Parana in the period from January 2006 to December 2012. The results showed that most of dogs $(56.25 \%)$ had weight bearing lameness on initial presentation. The unilateral RLCCr was the most frequent $(90.62 \%)$, $13.8 \%$ of these cases presented RLCCr in the contralateral limb, after an average period of 2 years and 4 months. The most frequent breeds were Toy Poodle (21.9\%) and Pitbull (18.75\%); mixed breed dogs accounted for $12.5 \%$ of cases. The most frequent ages were 2, 3, 4 and 7 years (average $=5.7$ years). With respect to sex and reproductive status, females (68.75\%) and non-castrated animals (69\%) were most affected. As for diet, the majority (59.38\%) ate commercial dog food. Regarding body weights, the majority ( $25 \%$ ) weighed between 26 and $35 \mathrm{~kg}$. DAD and patellar luxation prior to RLCCr were seen in $53.12 \%$ and $21.88 \%$ of the patients, respectively. It could be observed that the data in our environment tend to be similar to those of the literature: RLCCr occured in large adult dogs, weighing between 26 and $35 \mathrm{~kg}$ with unilateral rupture and weight bearing lameness and affected females more than males. Animals receiving commercial dog food and entire dogs were the most affected. Patellar luxation before RLCCr occurred in a minority of animals, different from the DAD preceding RLCCr, that was seen in most cases.
\end{abstract}

Key words: Claudication, degenerative joint disease, osteoarthritis, knee

\section{Introdução}

O joelho é uma complexa articulação ginglimoide, possuindo duas articulações funcionalmente distintas. A sustentação do peso ocorre principalmente por meio da articulação femorotibial, enquanto que a articulação femoropatelar aumenta significativamente a eficácia mecânica do grupo muscular extensor (VASSEUR, 2007).

O ligamento cruzado cranial que tem origem no interior da fossa intercondilar do fêmur, a partir da porção caudomedial do côndilo lateral, estendese na diagonal até a área intercondilar da tíbia (KÖNIG; LIEBICH, 2011). Este ligamento tem a função de restringir a articulação do joelho para impedir a hiperflexão e limitar a rotação interna e o deslocamento cranial da tíbia em relação ao fêmur (SLOCUM; SLOCUM, 1998; PIERMATTEI; FLO; De CAMP, 2009). De acordo com Mattiello-Rosa et al. (2008), lesão neste ligamento pode trazer graves sequelas para a articulação do joelho, incluindo comprometimento da estabilidade estática, alteração cinemática e da função motora, desencadeando sobrecarga articular e microlesões à cartilagem articular.

Muito foi descoberto sobre a ruptura do ligamento cruzado cranial (RLCCr), desde o primeiro relato feito em 1926 (ARNOCZY, 1985); mesmo assim, a causa da ruptura é, muitas vezes, desconhecida e o método terapêutico ideal permanece controverso (SCHULZ, 2008; TORRES; MUZZI; MESQUITA, 2012). Independentemente da técnica utilizada, as maiores complicações pós-cirúrgicas são a diminuição da funcionalidade e progressão da doença articular degenerativa (DAD) da articulação fêmoro-tíbio-patelar (ELKINS et al., 1991; HURLEY; HAMMER; SHOTT, 2007; TORRES; MUZZI; MESQUITA, 2012).

A RLCCr está frequentemente associada à claudicação aguda do membro pélvico 
e do desenvolvimento de DAD (DENNY; BUTTERWORTH, 2006; CANAPP JÚNIOR, 2007). De acordo com Vasseur (2007) e Piermattei, Flo e De Camp (2009) na maioria dos cães a ruptura tem curso crônico e não há um evento traumático distinto associado ao início do processo.

A RLCCr é frequentemente bilateral, já que de 30 a 40\% dos cães que apresentam RLCCr rompem o ligamento oposto em dois anos (PIERMATTEI; FLO; De CAMP, 2009).

Os cães de grande porte estão mais predispostos a RLCCr do que as raças de pequeno porte; as raças Rottweiler, Bull Mastiff e Chow Chow estão particularmente em risco (VASSEUR, 2007).

A RLCCr pode acontecer por causas agudas (traumáticas), que acometem mais comumente cães com menos de 4 anos de idade ou por causas crônicas (degenerativas), que acomete mais comumente cães entre 5 a 7 anos de idade. O enfraquecimento do ligamento pela degeneração permite que ele se rompa mais facilmente quando submetido a um exercício físico intenso (SCHULZ, 2008; WILKE, 2010).

Segundo Vasseur (2007) as fêmeas apresentam maior incidência de RLCCr que os machos e a castração contribui para o aparecimento da doença em ambos os sexos.

O nutriente que mais eleva o teor energético é a gordura, que por sua vez é melhor digerida. A gordura independente do tipo de alimento favorece o ganho de peso. O excesso de peso sobre os cães induz a RLCCr (PEIXOTO et al., 2010).

O diagnóstico clínico pode ser feito por meio dos testes de gaveta cranial e de compressão tibial, que permitem comprovar a translação cranial da tíbia. Como exame complementar recomenda-se radiografia para avaliar se está ocorrendo DAD (CANAPP JÚNIOR, 2007; OLIVEIRA et al., 2009). A tomografia computadorizada representa uma boa alternativa não só para o diagnóstico da RLCCr, mas também para a identificação das afecções associadas como lesões meniscais, lesões de superfície articular e osteofitose (KEALY; MCALLISTER; GRAHAM, 2011). A ressonância nuclear magnética é altamente sensível e específica na detecção de mínimas lesões à cartilagem articular e meniscos, além de estruturas livres no espaço articular, osteofitose e espessamento sinovial (SOLER et al., 2007).

Assim como o ligamento cruzado cranial, o ligamento patelar também é responsável pelo bloqueio da translação cranial da tíbia, razão pela qual quando há luxação patelar há predisposição para RLCCr (DENNY; BUTTERWORTH, 2006; SCHULZ, 2008; TORRES; MUZZI; MESQUITA, 2012). A luxação patelar causa instabilidade na articulação do joelho e DAD. Com o tempo a cartilagem articular da crista ou da patela sofre degeneração e o osso subcondral é exposto, causando dor significativa e agravamento do quadro degenerativo, fator também predisponente para a RLCCr (DENNY; BUTTERWORTH, 2006; SCHULZ, 2008; PIERMATTEI; FLO; De CAMP, 2009).

Preocupados em caracterizar a população de risco para a RLCCr, pesquisadores vêm tentando demonstrar, pelo estudo de amostras representativas de cães portadores da afecção, diversos fatores atrelados à ruptura (LAMPMAN; LUND; LIPOWITZ, 2003; MATERA et al., 2007).

O presente trabalho objetiva caracterizar a população de cães com RLCCr, atendidos na Unidade Hospitalar de Animais de Companhia (UHAC) da Pontifícia Universidade Católica do Paraná (PUCPR) de acordo com os fatores: tipo de claudicação no momento da consulta, bilateralidade, raça, idade, sexo, estado reprodutivo, dieta, peso corporal, presença de doença articular degenerativa (DAD) e/ou luxação patelar anteriores à RLCCr.

\section{Material e Métodos}

Foram analisados os prontuários de 32 cães atendidos na UHAC da PUCPR em um período 
de 7 anos (2006 a 2012), com o diagnóstico de RLCCr. Para o diagnóstico, os animais foram avaliados quanto ao histórico, tipo de claudicação, e foram realizados os testes de gaveta cranial e de compressão tibial. Como exame complementar foi realizada análise radiográfica.

O tipo de claudicação foi classificado como de apoio ou de elevação (nenhum dos coxins toca o chão), sendo esta última considerada como perda funcional do membro (VASSEUR, 2007).

Para a realização do teste de gaveta cranial, coloca-se o dedo indicador de uma mão sobre a patela e o polegar pressionando o côndilo lateral do fêmur; com outra mão, fixa-se a tíbia, colocando o dedo indicador na tuberosidade tibial e o polegar na cabeça da fíbula. Dessa maneira, com os dedos em forma de pinça e o membro semiflexionado testa-se o possível deslocamento cranial da tíbia em relação ao fêmur que indica a RLCCr (CANAPP JÚNIOR, 2007; SCHULZ, 2008). No teste da compressão tibial, fixa-se o joelho com uma das mãos, colocando o dedo indicador sobre a patela até tocar com a ponta do dedo a tuberosidade tibial. Com a outra mão, mantendo a extremidade semiflexionada, realizamse flexões do tarso. Ao realizar essas flexões, a tensão que se produz no músculo gastrocnêmio empurra a tíbia em sentido cranial, de forma que, observa-se o deslocamento do dedo situado sobre a crista tibial se houver RLCCr (DENNY; BUTTERWORTH, 2006; TORRES; MUZZI; MESQUITA, 2012).

Os animais foram avaliados quanto à ruptura uni ou bilateral, raça, idade, sexo, estado reprodutivo, dieta, peso corporal, presença de DAD e luxação patelar anteriores a RLCCr.

Para o cálculo da predisposição racial foi feita a separação das 32 fichas clinicas, que também serviram para a estatística etária. Para obtermos a média de idades dos animais, foi realizada a soma das idades depois divisão pelo total de raças.
Em relação ao sexo neste estudo participaram 22 fêmeas e 10 machos e o estado reprodutivo foi considerado, fêmeas castradas e não castradas e machos castrados e não castrados.

$\mathrm{O}$ tipo de dieta foi estimado quanto à administração de dieta comercial, dieta caseira e mista (associação de ambas).

Os cães foram agrupados segundo o peso em $<5$; 6 a15; 16 a 25; 26 a 35; 36 a 45 e $>46$ Kg. Segundo Hoskins (2008) cães de pequeno porte são aqueles que têm peso de até $9 \mathrm{~kg}$, cães de médio porte pesam de 9,5 a $22,5 \mathrm{~kg}$, cães de grande porte pesam de 23 a 40,5 kg e raças gigantes pesam acima de 40,5 kg.

A presença de DAD foi estimada após avaliações radiográficas das articulações com teste de gaveta cranial e/ou teste de compressão tibial positivas para RLCCr.

O registro das luxações patelares ocorreu no momento do exame ortopédico. Com o joelho semiflexionado o avaliador procurava deslocar com o seu polegar a patela lateral ou medialmente, deslocando-a para fora da tróclea femoral. Caso ocorresse o deslocamento, era registrada a luxação patelar como lateral ou medial.

\section{Resultados}

Dos 32 cães avaliados, a RLCCr ocorreu no joelho esquerdo de 15 (46,88\%), e no joelho direito de 14 (43,75\%), sendo bilateral no momento da consulta em apenas 3 (9,37\%). Percebeu-se que $56,25 \%$ dos animais apresentavam claudicação de apoio no momento da consulta, e 90,62\% RLCCr unilateral; destes, 13,79\% apresentaram ruptura no membro contralateral, em média após dois anos e quatro meses. A Tabela 1 mostra dados referentes ao padrão racial de cães portadores de RLCCr, enquanto que a Tabela 2 mostra dados referentes aos números absolutos e porcentagens de idades. 
Tabela 1. Dados referentes ao padrão racial de 32 cães portadores de RLCCr atendidos na UHAC da PUCPR no período de 2006 a 2012.

\begin{tabular}{lcc}
\hline Raça & Número absoluto (N) & Porcentagem Absoluta (\%) \\
\hline Poodle miniature & 7 & 21,9 \\
Pitbull & 6 & 18,75 \\
Rottweiler & 4 & 12,5 \\
SRD* & 4 & 12,5 \\
Bulldog & 3 & 9,38 \\
Weimaraner & 2 & 6,25 \\
Maltês & 1 & 3,12 \\
Labrador Retriever & 1 & 3,12 \\
Dogue Alemão & 1 & 3,12 \\
Cane Corso & 1 & 3,12 \\
Pinscher & 1 & 3,12 \\
Lhasa Apso & 1 & 3,12 \\
\hline Total & 32 & 100 \\
\hline
\end{tabular}

*SRD: Sem Raça Definida.

Fonte: Elaboração dos autores.

Tabela 2. Dados referentes aos números absolutos e porcentagens de idades dos 32 cães portadores de RLCCr atendidos na UHAC da PUCPR no período de 2006 a 2012.

\begin{tabular}{ccc}
\hline Idade (Anos) & Números Absolutos (N) & Porcentagem Absoluta (\%) \\
\hline 1 & 3 & 9,37 \\
2 & 4 & 12,5 \\
3 & 4 & 12,5 \\
4 & 4 & 12,5 \\
5 & 2 & 6,25 \\
6 & 2 & 6,25 \\
7 & 4 & 12,5 \\
8 & 1 & 3,13 \\
9 & 2 & 6,25 \\
10 & 3 & 9,37 \\
11 & 1 & 3,13 \\
12 & 0 & 0 \\
13 & 0 & 0 \\
Total & 2 & 6,25 \\
\hline
\end{tabular}

Fonte: Elaboração dos autores.

As fêmeas $(68,75 \%)$ foram mais representativas do que machos $(31,25 \%)$.

Os dados pertinentes ao sexo e estado reprodutivo, revelam maior incidência em fêmeas não castradas, como representado na Figura 1.

Dentre os animais estudados, 19 (59,38\%) ingeriram ração comercial, 13 (40,62\%) uma dieta mista, composta de ração comercial e dieta caseira. Nenhum deles recebia dieta exclusivamente caseira.

Os valores referentes ao peso corpóreo dos animais analisados encontram-se no Figura 2. Foi constada preponderância da ruptura em animais que pesavam entre 26 e $35 \mathrm{~kg}$. 
Figura 1. Distribuição dos 32 cães portadores de RLCCr, segundo o sexo e estado reprodutivo. Machos inteiros ( $\mathrm{n}=$ 9), machos castrados $(n=1)$, fêmeas inteiras $(n=13)$ e fêmeas castradas $(n=9)$.

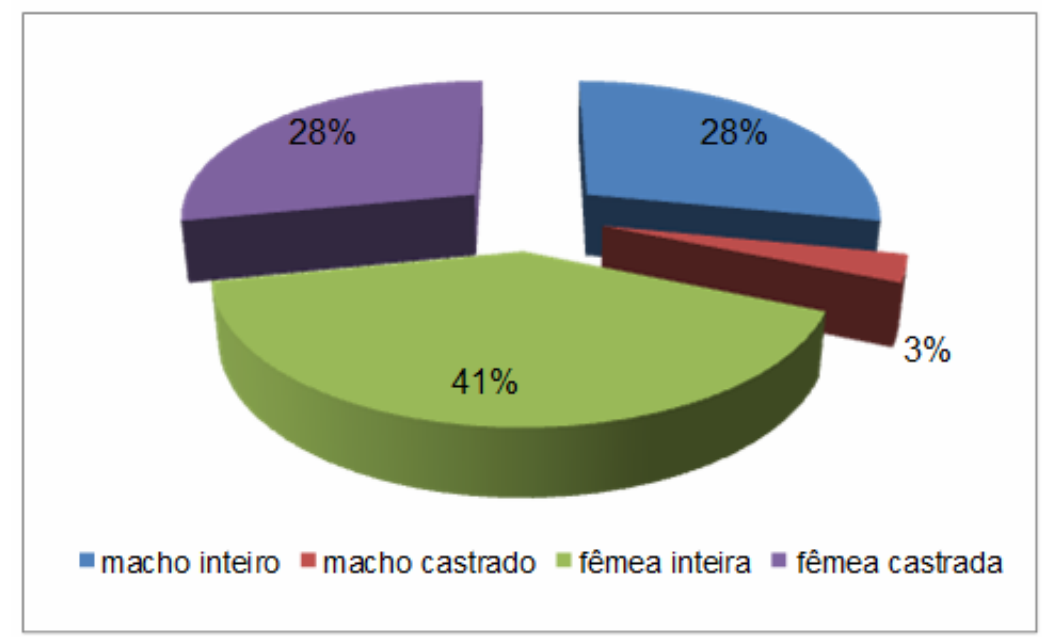

Fonte: Elaboração dos autores.

Figura 2. Gráfico de distribuição dos 32 cães com RLCCr, segundo o peso corpóreo: $<5 \mathrm{Kg}(\mathrm{n}=5), 6-15 \mathrm{~kg}(\mathrm{n}=7)$, $16-25(\mathrm{n}=5), 26-35 \mathrm{~kg}(\mathrm{n}=8), 36-45 \mathrm{~kg}(\mathrm{n}=6),>46 \mathrm{~kg}(\mathrm{n}=1)$.

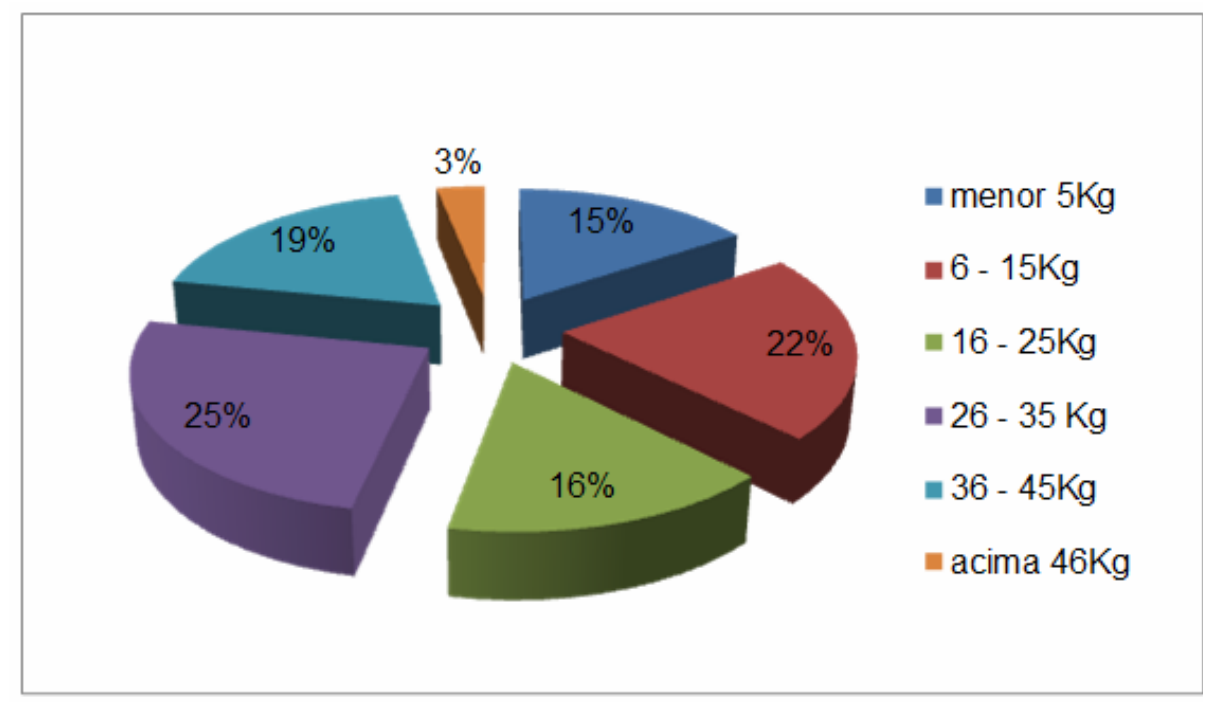

Fonte: Elaboração dos autores.

Foi constatada no momento da consulta a presença de sinais radiográficos de DAD em 17 cães $(53,12 \%)$. Os sinais radiográficos observados foram: deslocamento cranial da epífise proximal da tíbia em relação ao fêmur, aumento da radiopacidade do espaço articular, osteofitose, e avulsão óssea da superfície onde o ligamento se insere. A figura 3 representa um dos 32 casos avaliados, nela é possível observar proliferação óssea no sesamóide, proliferação óssea intra-articular e esclerose subcondral na epífise proximal da tíbia. 
Figura 3. Radiografia da articulação fêmoro-tíbio-patelar de cão na projeção mediolateral. Nota-se proliferação óssea no sesamóide (seta azul), proliferação óssea intra-articular (seta vermelha) e esclerose subcondral na epífise proximal da tíbia (seta amarela).

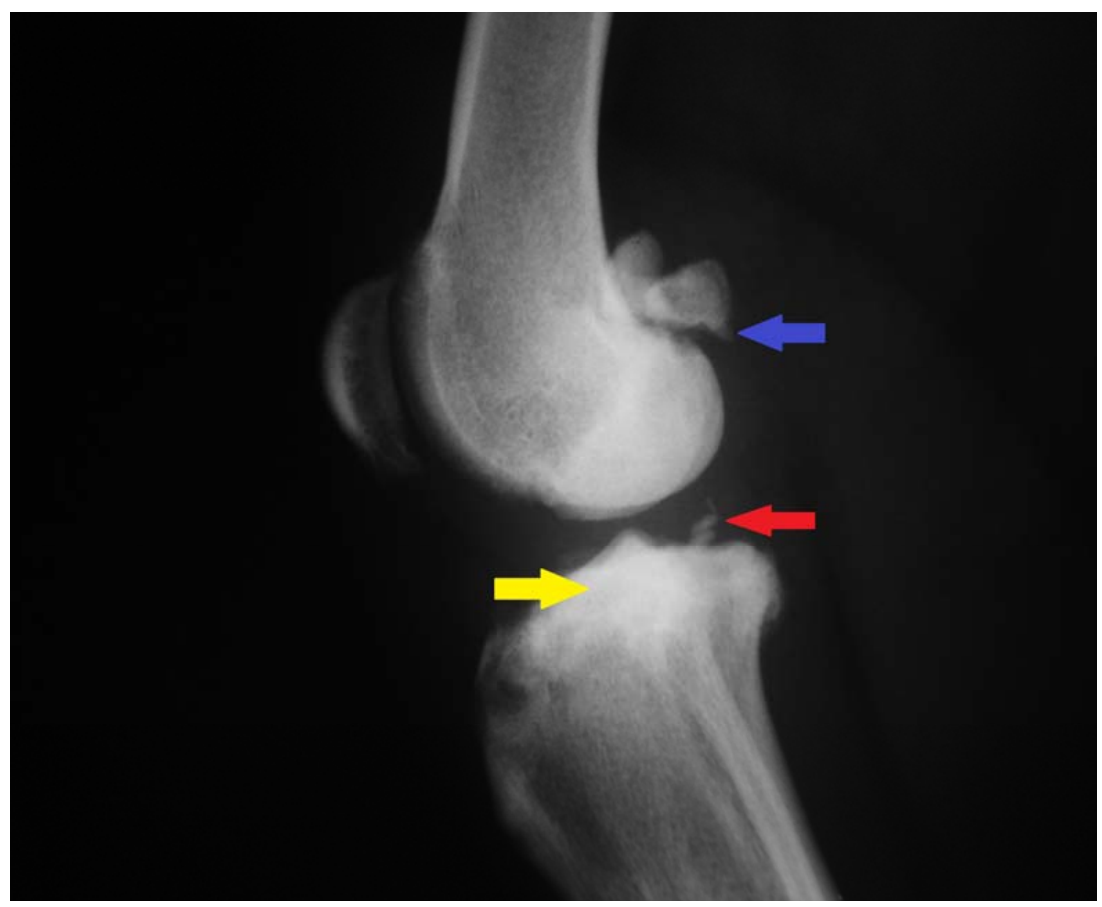

Fonte: Elaboração dos autores.

Nove $(28,13 \%)$ dos 32 cães com RLCCr apresentaram no momento da consulta luxação patelar, sendo $7(21,88 \%)$ medial e $2(6.25 \%)$ lateral.

\section{Discussão}

O prévio conhecimento dos fatores considerados de risco para uma determinada doença pode permitir melhor entendimento da sua etiologia, bem como fornecer informações sobre a sua prevenção e diagnóstico. Whitehair, Vasseur e Willits (1993) fizeram estudo epidemiológico e avaliaram a prevalência da idade, o tipo de dieta e a condição corpórea. Duval et al. (1999) e Lampman, Lund e Lipowitz (2003) procuraram caracterizar os cães portadores de RLCCr em relação à raça, sexo e peso corpóreo. Matera et al. (2007) por sua vez, caracterizaram os cães portadores de RLCCr quanto aos fatores considerados de risco como raça, idade, sexo, peso, dieta e padrão racial. Neste estudo procurou-se caracterizar os cães portadores de RLCCr atendidos na UHAC da PUCPR quanto aos fatores considerados de risco por Matera et al. (2007) e incluímos o tipo de claudicação no momento da consulta, se o acometimento era uni ou bilateral, a presença de sinais radiográficos de DAD e/ou luxação patelar anteriores a RLCCr.

Os dados oriundos deste trabalho foram comparados basicamente com a literatura norteamericana, salientando-se que a carência de trabalhos semelhantes em nosso meio motivou a sua realização.

As alterações degenerativas no ligamento são processos do envelhecimento natural, desenvolvidos em todos os cães. A resistência do ligamento cruzado cranial do cão declina com o envelhecimento; essa queda na resistência correlaciona-se com a perda da organização dos feixes de fibras colágenas e as alterações metaplásicas dos elementos celulares. Essas alterações são mais pronunciadas e ocorrem mais precocemente em cães de grande porte 
(BOSKEY, 2007), fator que foi constatado neste estudo, pois houve predomínio de lesão em cães com peso entre 26 a $35 \mathrm{~kg}$.

Ao se considerar a frequência em que um determinado padrão racial apresentou a RLCC, torna-se difícil a comparação dos resultados deste estudo com a literatura estrangeira, pois várias raças consideradas predisponentes para a afecção, como por exemplo, o Newfoundland e Starffordshire Terrier (LAMPMAN; LUND; LIPOWITZ, 2003), não são comuns em nosso meio. Outro fator conflitante relaciona-se ao modismo da raça em certo local e período de tempo (BENNETT; MAY, 1997; MATERA et al., 2007), tal qual se creditou a frequência da raça Pit Bull nesta pesquisa, a qual compreendeu $6(18,75 \%)$ animais e não foi citada na maioria dos trabalhos estudados.

As lesões do ligamento cruzado cranial têm sido classificadas como traumáticas ou degenerativas. Enquanto a primeira acomete animais em crescimento e ocorre avulsão de uma das extremidades do ligamento, na forma crônica a RLCC é espontânea, havendo um estiramento inicial, ruptura parcial e então total do ligamento (BENNETT; MAY, 1997). Os casos aqui relatados referem-se à forma crônica em sua maioria $(53,12 \%)$, nos quais havia a presença de sinais radiográficos de $\mathrm{DAD}$, sem relato de trauma pelo proprietário. Tanto a forma crônica, quanto a forma aguda estiveram presentes nas diferentes idades, sem predomínio evidente.

Harasen (2003) e Matera et al. (2007) relataram maior incidência da RLCCr em fêmeas do que em machos, fato constatado neste estudo. Porém, outros autores inferiram que animais castrados são mais susceptíveis a RLCCr do que animais não castrados (WHITEHAIR; VASSEUR; WILLITS, 1993; DUVALet al., 1999) e tal fato tem sido correlacionado com um estudo experimental realizado em ratas, nas quais a ovariectomia reduziu o conteúdo de elastina e o diâmetro das fibras colágenas da cápsula de articulação coxofemoral, justificando desta forma a ação dos hormônios sexuais no metabolismo do colágeno (SHIKATA; SANADA; YAMAMOTO, 1979). Os resultados deste estudo estão de acordo com os autores supracitados no que permeia ao maior acometimento de fêmeas quando comparado aos machos, porém a RLCCr foi mais frequente em animais não castrados do que em animais castrados.

Lampman, Lund e Lipowitz (2003) avaliaram a relação do tipo de dieta com a RLCCr no cão e concluíram que animais alimentados com dieta enriquecida e, consequentemente maior massa corporal foram mais representativos. A obesidade é citada como fator predisponente para a RLCCr no cão por promover carga excedente em um ligamento que já pode estar comprometido pelo processo degenerativo. Neste estudo, a grande parte dos cães recebia ração comercial, porém não tem informação sobre qual tipo e nem se os animais encontravam-se ou não acima do peso ideal.

Se avaliarmos a frequência de RLCCr quanto ao intervalo de peso corpóreo, notamos que esta foi maior em cães de 26 e $35 \mathrm{Kg}$ (25\%) seguido por animais entre 6 e $15 \mathrm{Kg}(21,89 \%)$, o que pode estar mais relacionado às raças (Poodle Toy e Pitbull, por exemplo), e não propriamente, com o excesso de peso.

A avaliação radiográfica da articulação do joelho pode ser um método auxiliar na confirmação da DAD. A projeção mediolateral em flexão de $90^{\circ}$ é a mais indicada para a visualização do deslocamento cranial da tíbia. O tarso deve estar flexionado, produzindo-se o mesmo efeito obtido no teste da compressão tibial. (OLIVEIRA et al., 2009; TORRES; MUZZI; MESQUITA, 2012).

A luxação patelar medial é muito mais comum que a luxação lateral em cães de todas as raças, representado 75 a $80 \%$ dos casos, com envolvimento bilateral observado em 20 a $25 \%$. A ruptura concomitante do ligamento cruzado cranial está presente em 15 a 20\% dos joelhos de cães de meiaidade com luxação patelar crônica (PIERMATTEI; FLO; De CAMP, 2009). Neste caso o ligamento 
cruzado cranial está submetido a grande tensão, visto que o mecanismo do quadríceps não é eficaz na estabilização da articulação (WILKE, 2010). Neste estudo houve maior incidência de luxação patelar medial e ruptura de ligamento cruzado unilateral, resultados que corroboram os trabalhos estudados.

Apesar da causa da RLCCr ser ainda desconhecida, achados importantes têm sido obtidos em estudos realizados. Diferentes propriedades físicas do ligamento cruzado cranial entre as raças, estenose do sulco intercondilar, diferentes ângulos do platô tibial e da conformação do membro pélvico, além de DAD e/ou luxação patelar anteriores a RLCCr têm sido imputados como prováveis fatores predisponentes para a afecção (DUVAL et al., 1999; GUERRERO et al., 2007; OLIVEIRA et al., 2009; DRYGAS et al., 2010; KIM et al., 2010; KEALY; MCALLISTER; GRAHAM, 2011). A correlação destas alterações com as diferentes raças poderem contribuir com a prevenção e terapêutica adequadas.

\section{Conclusões}

Por meio deste trabalho pode-se observar que os dados coletados se assemelham com a literatura nacional e estrangeira. Na maioria das vezes não há perda funcional, existindo maior manifestação unilateral. As raças com maior incidência segundo este estudo foram poodle e pitbull. A maioria dos animais acometidos de RLCCr, eram jovens do sexo feminino, não castrados. A dieta comercial e o peso entre 26-35 kg foram os dados mais frequentemente observados nos animais deste estudo. Doença articular degenerativa preexistente à RLCCr foi vista na maioria dos animais. Apesar da maioria dos cães presentes neste estudo não apresentarem luxação patelar anterior à RLCCr, consideramos a luxação patelar, seja ela medial ou lateral, como importante fator predisponente à RLCCr.

Acreditamos que estes dados são importantes fatores atrelados à RLCCr e que servirão para situar os padrões de ocorrência da lesão; haja vista que esta afecção é uma das lesões mais comuns no cão e a maior causa de doença articular degenerativa do joelho.

\section{Referências}

ARNOCZY, S. P. Cruciate ligament rupture and associate injuries: In: NEWTON, C. D.; NUNAMAKER, D. M. Textbook of small animall orthopaedics. Philadelphia: Lippincott, 1985. p. 403-414.

BENNET, D.; MAY, C. Moléstias articulares de cães e gatos. In: ETTINGER, S. J.; FELDMAN, E. C. Tratado de medicina interna. 4. ed. São Paulo: Manole, 1997. p. 2805-2866.

BOSKEY, A. L. Tecidos conjuntivos do sistema musculoesquelético. In: SLATTER, D. Manual de cirurgia de pequenos animais. 3. ed. São Paulo: Manole, 2007. p. 1774-1784.

CANAPP JÚNIOR, S. O. The canine stifle. Clinical Techniques in Small Animal Practice, St. Louis, v. 22, n. 4, p. 195-205, 2007.

DENNY, H. R.; BUTTERWORTH, S. J. Cirurgia ortopédica em cães e gatos. 4. ed. São Paulo: Roca, 2006. p. 396-427.

DRYGAS, K. A.; POZZI, A.; GORING, R. L.; HORODYSKI, M.; LEWIS, D. D. Effect of tibial leveling osteotomy on patellar tendon angle: a radiographic cadaver study. Veterinary Surgery, Hoboken, v. 39, n. 4, p. 418-424, 2010.

DUVAL, J. M.; BUDSBERG, S. C.; FLO, G. L.; SAMMARCO, J. L. Breed, sex, and body weight as risk factors for rupture of the cranial cruciate ligament in young dogs. Journal of American Veterinary Medical Association, Illinois, v. 215, n. 6, p. 811-814, 1999.

ELKINS, A. D.; PECHMAN, R.; KEARNEY, M. T. HERRON, M. A retrospective study evaluating the degree of degenerative joint disease in the stifle joint of dogs following surgical repair of anterior cruciate ligament rupture. Journal of the American Animal Hospital Association, Lakewood, v. 27, n. 1, p. 533-540, 1991.

GUERRERO, T. G.; GEYER, H.; HASSIG, M.; MONTAVON, P. M. Effect of conformation of the distal portion of the femur and proximal portion of the tibia on the pathogenesis of cranial cruciate ligament disease in dogs. American Journal of Veterinary Research, Chicago, v. 68, n. 12, p. 1332-1337. 2007.

HARASEN, G. Canine cranial cruciate ligament rupture in profile. Canine Veterinary Journal, Bethesda, v. 44, n. 5, p. 845-846, 2003. 
HOSKINS, J. D. Geriatria e gerontologia do cão e do gato. 2. ed. São Paulo: Ed. Roca, 2008. 448 p.

HURLEY, C. R.; HAMMER, D. L.; SHOTT, S. Progression of radiographic evidence of osteoarthritis following tibial plateau leveling osteotomy in dogs with cranial cruciate ligament rupture: 295 cases (2001-2005). Journal of American Veterinary Medical Association, Illinois, v. 230, n. 11, p. 1674-1679, 2007.

KEALY, J. K.; MCALLISTER, H.; GRAHAM, J.P. Bones and joints. Diagnostic radiology and ultrasonography of the dog and cat. 5. ed. St. Louis: Elsevier Saunders, 2011. p. 351-446.

KIM, S. E.; POZZI, A.; BANKS, S. A.; CONRAD, B. P.; LEWIS, D. D. Effect of cranial cruciate ligament deficiency, tibial plateau leveling osteotomy, and tibial tuberosity advancement on contact mechanics and aligment of the stifle in flexion. Veterinary Surgery, Hoboken, v. 39, n. 3, p. 363-370, 2010.

KÖNIG, H. E.; LIEBICH, H. Anatomia dos animais domésticos. 4. ed. São Paulo: Ed. Artmed, 2011. 788 p.

LAMPMAN, T. J.; LUND, E. M.; LIPOWITZ, A. J. Cranial cruciate disease: current status of diagnosis, surgery, and risk for disease. Veterinary Comparative Orthopedics and Traumatology, Stuttgart, v. 16, n. 3, p. 122-126, 2003.

MATERA, J. M.; TATARUNAS, A. C.; OLIVEIRA, R. M. D.; BRUGNARO, M.; MACCHIONE, R. F. Estudo epidemiológico retrospectivo de cães portadores de ruptura do ligamento cruzado cranial: 323 casos (1999 a 2005). Brazilian Journal of Veterinary Research and Animal Science, São Paulo, v. 44, n. 1, p. 88-95, 2007.

MATTIELLO-ROSA, S. M. G.; CINTRA NETO, P. F. A.; LIMA, G. E. G.; PINTO, K. N. Z.; COHEN, M.; PIMENTEL, E. R. Glycosaminoglycan loss from cartilage after Anterior Cruciate Ligament rupture: influence of time since rupture and chondral injury. Revista Brasileira de Fisioterapia, São Carlos, v. 12, n. 1, p. 64-69, 2008.

OLIVEIRA, R. R.; MAMPRIM, M. J.; RAHAL, S. C.; BICUDO, A. L. C. Radiologia e ultrassonografia no diagnóstico da ruptura do ligamento cruzado cranial em cães. Pesquisa Veterinária Brasileira, Rio de Janeiro, v. 29, n. 8, p. 661-665, 2009.
PEIXOTO, M.; BRUNETTO, M. A.; NOGUEIRA, S. P.; SÁ, F. C.; GOMES, M. O. S.; JEREMIAS, J. T.; CARCIOFI, A. C. Avaliação do perfil lipídico em cães obesos. In: CIC - CONGRESSO DE INICIAÇÃO CIENTÍFICA DA UNESP, 21., 2009, São José do Rio Preto. Anais... São José do Rio Preto: FUNEP, 2009. p. 424-427.

PIERMATTEI, D. L.; FLO, L.; De CAMP, C. E. A articulação do joelho. Ortopedia e tratamento de fraturas de pequenos animais. 4. ed. Barueri: Ed. Manoele, 2009. p. 637-717.

SCHULZ, K. Afecções articulares. In: FOSSUM, T. W. Cirurgia de pequenos animais. 3. ed. São Paulo: Roca, 2008. p. 1254-1276.

SHIKATA, J.; SANADA, H.; YAMAMOTO, T. Experimental studies of the elastic fiber of the capsular ligament: influence of aging and sex hormones on the hip and knee joint capsule of rats. Connect Tissue Research, London, v. 7, n. 1, p. 21-27, 1979.

SLOCUM, B.; SLOCUM, T. D. Algorithm for diagnosis and treatment of the stifle for cranial cruciate ligament rupture. In: BOJRAB, M. J.; ELLISON, G. W.; SLOCUM, B. Current techniques in small animal surgery. 4. ed. Baltimore: Lippincott Williams \& Wilkins, 1998. p. 1187-1193.

SOLER, M.; MURCIANO, J.; LATORRE, R.; BELDA, E.; RODRÍGUEZ, M. J.; AGUT, A. Ultrasonographic. computed tomographic and magnetic resonance imaging anatomy of the normal canine stifle joint. The Veterinary Journal, St. Louis, v. 174, n. 2, p. 351-361, 2007.

TORRES, B. B. J.; MUZZI, L. A. L.; MESQUITA L. R. Ruptura do ligamento cruzado cranial - revisão. Clínica Veterinária, São Paulo, v. 17, n. 100, p. 100-112, 2012.

VASSEUR, P. B. Articulação do joelho. In: SLATTER, D. Manual de cirurgia de pequenos animais. 3. ed. São Paulo: Manole, 2007. p. 2090-2133.

WHITEHAIR, J. G.; VASSEUR, P. B.; WILLITS, N. H. Epidemiology of cranial cruciate ligament rupture in dogs. Journal of American Veterinary Medical Association, Illinois, v. 203, n. 7, p. 1016-1019, 1993.

WILKE, V. Genetics of cranial cruciate ligament rupture. In: MUIR, P. Advances in the canine cranial cruciate ligament. Iowa: Wiley Blackwell, 2010. p. 53-58. 